\title{
VEHICLE SIDESLIP ANGLE ESTIMATION USING TWO SINGLE-ANTENNA GPS RECEIVERS
}

\author{
Jong-Hwa Yoon \\ Department of Mechanical Engineering \\ University of Michigan \\ Ann Arbor, MI 48109 \\ E-mail: jongy@umich.edu
}

\author{
Huei Peng \\ Department of Mechanical Engineering \\ University of Michigan \\ Ann Arbor, MI 48109
}

\section{ABSTRACT}

Knowing vehicle sideslip angle accurately is critical for active safety systems such as Electronic Stability Control (ESC). Vehicle sideslip angle can be measured through optical speed sensors, or dual-antenna GPS. These measurement systems are costly ( $\$ 5 k$ to $\$ 100 k)$, which prohibits wide adoption of such systems. This paper demonstrates that the vehicle sideslip angle can be estimated in real-time by using two low-cost single-antenna GPS receivers. Fast sampled signals from an Inertial Measurement Unit (IMU) compensate for the slow update rate of the GPS receivers through an Extended Kalman Filter (EKF). Bias errors of the IMU measurements are estimated through an EKF to improve the sideslip estimation accuracy. A key challenge of the proposed method lies in the synchronization of the two GPS receivers, which is achieved through an extrapolated update method. Analysis reveals that the estimation accuracy of the proposed method relies mainly on vehicle yaw rate and longitudinal velocity. Experimental results confirm the feasibility of the proposed method.

\section{NOMENCLATURE}

$\mathrm{U}, \mathrm{V} \quad$ Longitudinal and lateral velocities (in vehicle frame)

$\psi, \dot{\psi} \quad$ Heading angle and yaw rate

$V_{f e}, V_{f n} \quad$ Eastbound and Northbound velocities of the GPS receiver located on the front end of the vehicle

$V_{r e}, V_{r n} \quad$ Eastbound and Northbound velocities of the GPS receiver located on the rear end of the vehicle

$a_{x m}, a_{y m}$ Measured longitudinal and lateral accelerations at center of mass $r_{m} \quad$ Measured yaw rate

$L_{f}, L_{r}, L$ Distance between the front GPS receiver and vehicle center of mass, between the rear GPS receiver and vehicle center of mass, and between the two GPS receivers, respectively

$x_{k}, z_{k} \quad$ State vector and measurement at step $\mathrm{k}$

$\hat{x}_{m \mid n} \quad$ State estimation at step m based on measurement up to step $n$

$P_{m \mid n} \quad$ State estimation covariance matrix at step $\mathrm{m}$ based on measurement up to step $n$

$Q_{k} \quad$ Disturbance covariance matrix at step k

$\tilde{y}_{k} \quad$ Measurement residual at step $\mathrm{k}$

$K_{k} \quad$ Optimal Kalman Filter gain at step k

$R_{k} \quad$ Measurement noise covariance matrix at step k

$f, h \quad$ State transition function and measurement function

$b_{x}, b_{y}, b_{r}$ Biases of longitudinal/lateral accelerometer and rate gyro

$w_{x}, w_{y}, w_{r}$ White noise in longitudinal/lateral accelerometer and rate gyro

$T \quad$ Delay time in GPS velocity measurement

H.O.T Higher Order Terms 


\section{INTRODUCTION}

Sideslip angle is an important state of vehicle lateral dynamics, and its estimation has been studied by many researchers [1-10]. Existing methodologies can be categorized into three groups: Kinematics-based estimation, kinetic modelbased estimation, and GPS-based measurements. Kinematicsbased method is robust to vehicle parameter errors and works well even when the sideslip angle is outside of the linear range of sideslip-lateral force relationship. However, it is sensitive to sensor bias and disturbances such as road inclination. Kinetic model-based methods require very accurate parameter information, and the small sideslip angle assumption is critical.

Bevly et al. proposed an estimation method utilizing a single antenna GPS along with inertial measurement unit (IMU) $[8,9]$. Basically this method integrates the bias-corrected yaw rate to obtain vehicle heading angle, which can provide the sideslip angle along with the vehicle velocity angle measured by the single antenna GPS. In spite of the originality of this method, the side slip estimation error will grow over time and stable yaw gyro bias and high accuracy of its estimation are required. These performance limits are inevitable to overcome the inherent limitation of algorithms using a single antenna GPS: the vehicle heading angle is unobservable with only a single GPS antenna [8]. The idea of utilizing multiple GPS receivers to measure sideslip angle was explored by researchers at the Ford Motor Company and the concept is described in a patent [11]. Although this method mainly utilizes the relationship among the velocities of multiple GPS receivers, the vehicle heading angle, which is very critical in sideslip estimation, is calculated by subtracting position vectors of GPS receivers. Since the position measurement of a today's low-cost GPS receiver has poor accuracy $(\sim 5 \mathrm{~m})$, advanced positioning technologies need to be employed. Ryu et al. also investigated the application of dual antenna GPS for sideslip measurement.[10] It has several advantages, including being vehicle parameter free, working at large sideslip angle, and providing accurate vehicle heading angle. However, the high cost of dual-antenna is a major roadblock for practical applications. With dual antenna GPS, the vehicle heading angle is calculated by measuring phase difference of the carrier waves received at the two antennas at known locations on the vehicle [12]. The phase difference detection capability is believed to be the main reason for high hardware cost today.

This paper presents a new method utilizing two low-cost single GPS antennas to estimate vehicle sideslip angle. Four kinematics equations which connect velocities of two different GPS receivers to the heading angle and longitudinal/lateral velocities of the vehicle are presented. Analysis of these equations is conducted to find the theoretical limit of the sideslip/heading angle estimation. GPS/IMU fusion through an Extended Kalman Filter is discussed. For an accurate GPS/IMU fusion, measurements from IMU and two GPS receivers must be aligned to represent concurrent data, called synchronization henceforth. One popular method for GPS/IMU synchronization is to tag IMU measurements with coordinated universal time with the aid of 1 pulse-per-second signal from GPS receiver. This method can facilitate tight synchronization but requires direct access to the data collection hardware [13]. Another method sets the unsynchronized time difference as a state and estimates it through an augmented Kalman Filter [14]. This method can be implemented without direct access to the hardware. This paper utilized the latter method for GPS/IMU synchronization. Direct synchronization between two GPS receivers was not tried. Instead, an extrapolation method was exploited to reduce the error caused by the unsynchronized updates between two GPS receivers. A small pick-up truck equipped with two single-antenna GPS receivers and an IMU (longitudinal/lateral acceleration and gyroscope) was used to conduct road test to verify the performance of the proposed method experimentally.

\section{KINEMATICS OF IMU AND TWO GPS RECEIVERS}

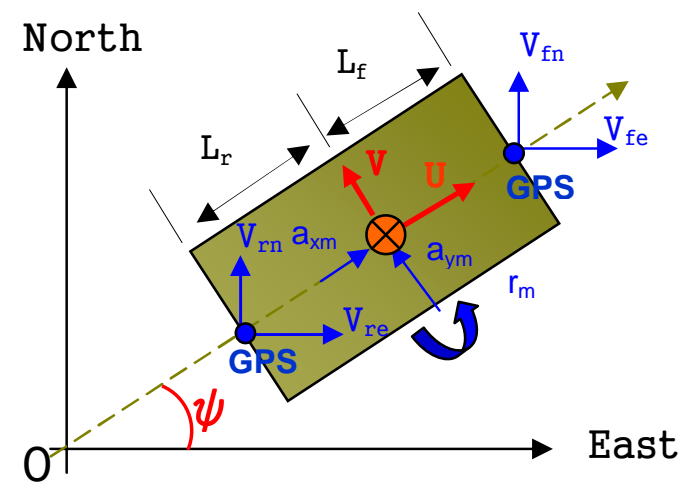

\section{FIGURE 1. TOP VIEW OF A VEHICLE ON THE PLANAR SURFACE}

Figure 1 shows the top view of a vehicle on the planar surface. Four GPS planar velocities (East/North velocities of Front/Rear GPS) can be expressed as functions of longitudinal/lateral velocities, yaw rate and heading angle:

$$
\begin{aligned}
V_{f e} & =U \cos \psi-\left(V+L_{f} \dot{\psi}\right) \sin \psi \\
V_{f n} & =U \sin \psi+\left(V+L_{f} \dot{\psi}\right) \cos \psi \\
V_{r e} & =U \cos \psi-\left(V-L_{r} \dot{\psi}\right) \sin \psi \\
V_{r n} & =U \sin \psi+\left(V-L_{r} \dot{\psi}\right) \cos \psi
\end{aligned}
$$

Although the number of equations and unknowns are the same, these equations are not necessarily solvable. Furthermore, the effect of GPS velocity measurement noise on the accuracy of longitudinal/lateral velocity and heading angle estimation needs to be analyzed. To find the singular condition when estimation of four states is impossible, and to understand how GPS velocity measurement errors propagate in states estimation calculations, the Taylor's Theorem was applied (Eq. (5)) 


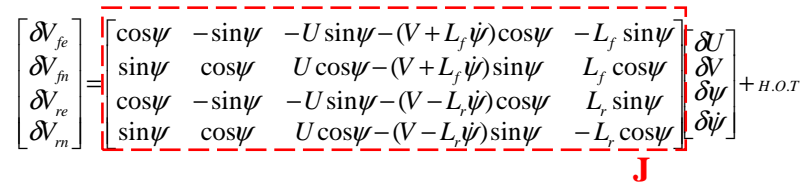

Ignoring the higher order terms, Eq. (5) becomes linear. Since the determinant of the Jacobian matrix is

$$
\operatorname{det}(J)=L^{2} \dot{\psi}
$$

Eq. (5) becomes singular if and only if the vehicle yaw rate is zero. Figure 2 gives an intuitive explanation why zero yaw rate corresponds to the singular situation. With zero yaw rate, velocity measurements of the two GPS receivers will be identical for the two cases A and B. Therefore, it is impossible to infer vehicle heading angle when yaw rate is zero. For heading angle calculations, velocities of the two GPS receivers need to be coupled with the heading angle through non-zero yaw rate.

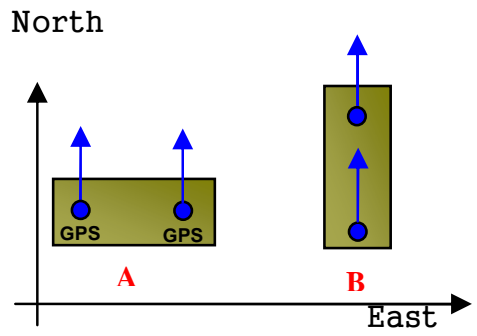

FIGURE 2. ZERO YAW RATE CORRESPONDS TO SINGULAR CONDITION

\section{Resolution Analysis}

When yaw rate is not zero, Eq.(5) is non-singular and change in the four states (longitudinal and lateral velocities, heading angle and yaw rate) can be obtained by inverting the Jacobian matrix :

$$
\left[\begin{array}{l}
\delta U \\
\delta V \\
\delta \psi \\
\delta \dot{\psi}
\end{array}\right]=J^{-1}\left[\begin{array}{l}
\delta V_{f e} \\
\delta V_{f n} \\
\delta V_{r e} \\
\delta V_{r n}
\end{array}\right]
$$

In addition, the standard deviation of a signal which is a linear combination of two other signals, assuming the two signals are uncorrelated, can be computed from

$$
\sigma^{2}(a X+b Y)=a^{2} \sigma^{2}(X)+b^{2} \sigma^{2}(Y)
$$

By applying Eq. (8) to Eq. (7) and assuming the standard deviations of the GPS horizontal velocities are identical, we have

$$
\begin{aligned}
& \sigma(\delta \psi)=\frac{\sqrt{2}}{|L \dot{\psi}|} \sigma\left(\delta V_{G P S}\right) \\
& \sigma(\delta \beta)=\frac{\sqrt{2 U^{2}+\left(L_{f}^{2}+L_{r}^{2}\right) \dot{\psi}^{2}}}{|L U \dot{\psi}|} \sigma\left(\delta V_{G P S}\right)
\end{aligned}
$$

where the vehicle sideslip angle $\beta$ is defined $\operatorname{astan}^{-1}(V / U)$. Error analysis of heading angle $(\psi)$ is derived because it is critical information for sideslip estimation. Figure 3 shows the numerical results of Eqs. (9), (10). It can be seen that the heading angle estimation accuracy is proportional to the accuracy of the GPS speed measurement, which is beyond our control. However, it can also be seen that the accuracy is inversely proportional to vehicle yaw rate and the distance between the two GPS receivers (L). Sideslip angle estimation becomes more accurate with higher yaw rate and high vehicle longitudinal velocity. Note that standard deviation of the sideslip angle error is close to but always higher than that of the heading angle. Table 1 presents theoretical limits of heading and sideslip angle estimation under the assumed white Gaussian noise of the GPS velocity measurement (standard deviation of the GPS receiver utilized in this paper is measured to be $0.01 \mathrm{~m} / \mathrm{s}$ ). In the calculations, the vehicle forward speed is assumed to be $10 \mathrm{~m} / \mathrm{s}$.
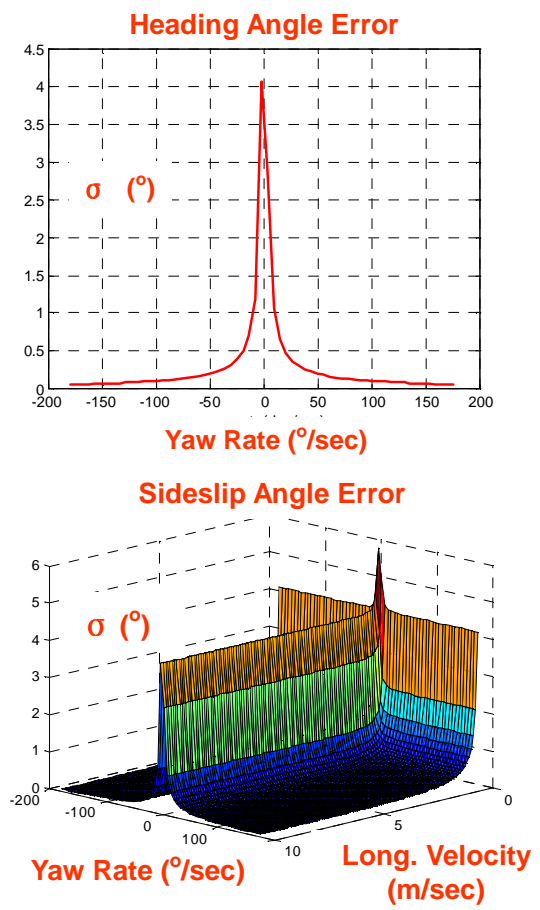

FIGURE 3. STANDARD DEVIATION OF THE HEADING ANGLE AND SIDESLIP ANGLE ESTIMATION UNDER WHITE GAUSSIAN GPS VELOCITY NOISE 
TABLE 1. THEORETICAL PERFORMANCE OF THE HEADING ANGLE AND SIDESLIP ANGLE ESTIMATION ERROR $(1 \sigma)$

\begin{tabular}{|c|c|c|c|}
\hline Yaw Rate $(\% / \mathrm{sec})$ & 15 & 30 & 45 \\
\hline Heading Angle $\left(^{\circ}\right)$ & 0.64 & 0.32 & 0.21 \\
\hline Sideslip Angle $\left(^{\circ}\right)$ & 0.65 & 0.33 & 0.22 \\
\hline
\end{tabular}

\section{GPS/IMU FUSION BY EXTENDED KALMAN FILTER}

To capture dynamic motions of the vehicle, a GPS receiver with an output rate of $5 \mathrm{~Hz}$ or higher should be used when possible [8]. However, the update rate of today's low-cost GPS receivers can be lower. Actually, the GPS receivers we purchased off the shelf have an update rate of $2.5 \mathrm{~Hz}$. To compensate for this slow update rate, GPS/IMU fusion is a possible option. The characteristics of IMU signals (high update rate but can have bias) are complementary to those of the GPS signals (low update rate, un-biased) and thus form an ideal pair for sensor fusion. Due to the non-linearity of Eqs. (1) (4), the Extended Kalman Filter technique is utilized for the sensor fusion. Typical Extended Kalman Filter(EKF) has a structure in the following [15].

$$
\begin{aligned}
& \hat{x}_{k \mid k-1}=f\left(\hat{x}_{k-1 \mid k-1}, u_{k-1}\right) \\
& P_{k \mid k-1}=\Phi_{k-1} P_{k-1 \mid k-1} \Phi_{k-1}^{T}+Q_{k-1} \\
& \tilde{y}_{k}=z_{k}-h\left(\hat{x}_{k \mid k-1}\right) \\
& K_{k}=P_{k \mid k-1} H_{k}^{T}\left(H_{k} P_{k \mid k-1} H_{k}^{T}+R_{k}\right)^{-1} \\
& \hat{x}_{k \mid k}=\hat{x}_{k \mid k-1}+K_{k} \tilde{y}_{k} \\
& P_{k \mid k}=\left(I-K_{k} H_{k}\right) P_{k \mid k-1} \\
& x_{k}=f\left(x_{k-1}, u_{k-1}\right)+w_{k-1} \\
& z_{k}=h\left(x_{k}\right)+v_{k} \\
& \Phi_{k-1}=\left.\frac{\partial f}{\partial x}\right|_{\hat{x}_{k-1 \mid k-1}, u_{k}} \\
& H_{k}=\left.\frac{\partial h}{\partial x}\right|_{\hat{x}_{k \mid k-1}}
\end{aligned}
$$

Measurement covariance $\left(R_{k}\right)$ in Eq. (14) sets the weight of the measurement for state updates (Eq. (15)). The smaller it is, the more impact the measurement has on the state update, and vice versa. Resolution analysis revealed that heading angle and sideslip angle measurement become more reliable as yaw rate increases. To consider this in EKF above, the measurement covariance $\left(R_{k}\right)$ is modified to decrease as yaw rate increases.

\section{Dynamics of the IMU Sensors}

IMU sensor outputs are related to the vehicle lateral, longitudinal and yaw motions in vehicle-fixed frame, and the governing equations are:

$$
\begin{aligned}
a_{x m} & =\dot{U}-V \dot{\psi}+b_{x}+w_{x} \\
a_{y m} & =\dot{V}+U \dot{\psi}+b_{y}+w_{y} \\
r_{m} & =\dot{\psi}+b_{r}+w_{r}
\end{aligned}
$$

The three bias terms $\left(b_{x}, b_{y}, b_{r}\right)$ of the IMU measurements are assumed to be constant throughout the measurement windows, i.e.,

$$
\dot{b}_{x}=0, \quad \dot{b}_{v}=0, \quad \dot{b}_{r}=0
$$

Using Eq. (21) (24), an augmented state-space equation is obtained

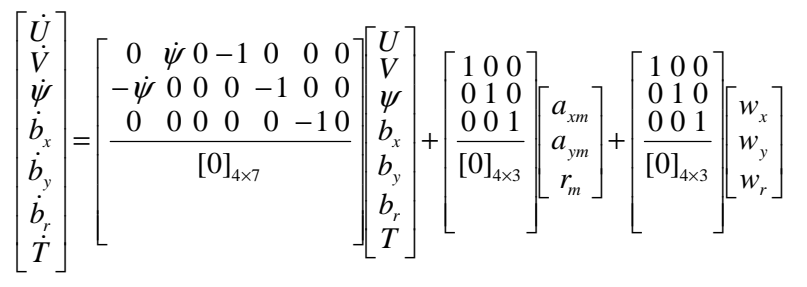

$A$ variable $T$ is the time difference between when the actual vehicle velocity occurs and the time when measurement is available from the GPS receiver. This delay exists because of the way to calculate the velocity, data processing time, and other communication delays exist in the overall sensing and estimation system. The estimated value of this variable will be used for better synchronization between the GPS and IMU signals. High frequency measurement noises also are approximated by Gaussian white noises and their covariance values are assumed to be known and described by the following equations [16].

$$
\begin{gathered}
Q_{c}^{\prime}=\varepsilon\left\{\bar{w} \bar{w}^{T}\right\}=\left[\begin{array}{ccc}
\sigma^{2}{ }_{w_{x}} & 0 & 0 \\
0 & \sigma^{2}{ }_{w_{y}} & 0 \\
0 & 0 & \sigma^{2}{ }_{w_{r}}
\end{array}\right] \\
Q_{k-1}=\int_{0}^{T_{s}} \Phi_{k-1}(\tau) \Gamma_{k-1} Q_{c}^{\prime} \Gamma^{T}{ }_{k-1} \Phi(\tau)^{T}{ }_{k-1} d \tau
\end{gathered}
$$

\section{GPS/IMU \& GPS/GPS Synchronization}

The unsynchronized updates between GPS and IMU were known to be major error sources [14]. The GPS receivers utilized for this paper estimate its velocity by interpreting the change in carrier phase between successive samples. This method is known to have inherent latency equal to one half of the sampling time in delivering velocity measurements [12]. Since the update frequency of the GPS receiver of this work is $2.5 \mathrm{~Hz}$, the latency is about $200 \mathrm{~ms}$. Moreover, the uncertain internal data processing time ( $<300 \mathrm{~ms}$ by specification) of the GPS module is added. Additionally, other possible communication delays may make nominal calculation of total 
delay more uncertain. Several methods were proposed in the literature for time synchronization among multiple sensors possibly including a GPS receiver. They can be categorized into hardware-based and software-based methods. In hardwarebased methods, one Pulse-Per-Second signal (PPS) output from a GPS receiver is used as the time-sync reference to other sensors. This method requires direct access to the hardware of the sensor modules and modification of the sensor software. In software-based method, the time latency between GPS and other sensors is estimated and used to correct the measurements. Since delay of IMU signal is negligible $(6 \mathrm{~ms})$ compared to GPS signal delay $(\sim 500 \mathrm{~ms})$, all IMU signals are treated as realtime information. Therefore, time difference between GPS and IMU can be treated same as the delay of GPS signals. The Kalman Filter method presented in the previous section falls into this category. Figure 4 shows a hypothetical case with the velocity measurement of a GPS receiver. The relation between the actual and measured vehicle speed is approximated by

$$
V_{(t)}^{G P S}=V_{(t-T)}^{\text {real }}=V_{(t)}^{\text {real }}-\frac{d V_{(t)}^{\text {real }}}{d t} T+H . O . T
$$

where $\mathrm{V}^{\text {real }}(\mathrm{t})$ is the true GPS speed at ' $\mathrm{t}$ ' and is related to vehicle motion variables $[U, V, \psi, \dot{\psi}]$. By assuming that $\ddot{\psi}$ is small, we have

$$
V_{(t)}^{G P S} \approx V_{(t)}^{\text {real }}-\left(\frac{\partial V^{\text {real }}}{\partial U} \frac{d U}{d t}+\frac{\partial V^{\text {real }}}{\partial V} \frac{d V}{d t}+\frac{\partial V^{\text {real }}}{\partial \psi} \frac{d \psi}{d t}\right) T
$$

By combining Eq. (1) (4) and Eq. (29), the measurement function $h$ in Eq. (18) is obtained.

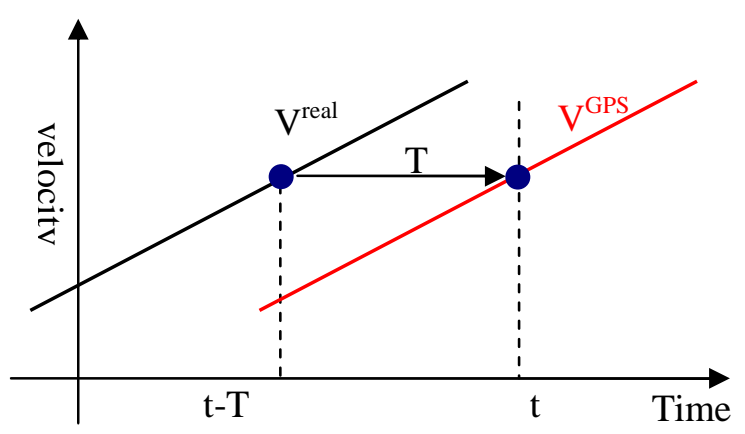

FIGURE 4. TIME DELAY IN GPS MEASUREMENT

When multiple GPS receivers are used, the unsynchronized measurements from these GPS units will further deteriorate the performance of vehicle speed estimation. The concept of unsynchronized GPS measurements is illustrates in Figure 5.

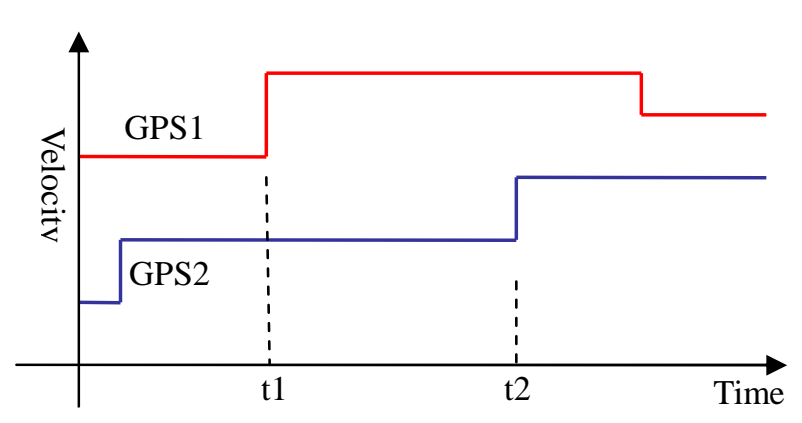

\section{FIGURE 5. UNSYNCHRONIZED UPDATES FROM TWO GPS RECEIVERS}

At time $\mathrm{t} 2$, the measurement update from GPS2 is obtained and the Kalman Filter update is to be obtained. However, since the measurement from GPS1 is from time $t 1$, it is "old" and if mixed with the measurements from GPS2, erroneous side slip estimates will be obtained. The latency between $\mathrm{t} 1$ and $\mathrm{t} 2$ can reach one full sampling period of the GPS receiver ( $400 \mathrm{~ms}$ for this work), which is unacceptable. To solve this problem, a 'Quadratic Extrapolation Method' is proposed. Figure 6 explains the concept of this method. The Kalman filter is to be updated at $\mathrm{t} 2$, which requires accurate measurements at that time instant. Assume that the last update from a GPS receiver occurred at time $\mathrm{t} 1$. Then, three historical values from that GPS are used to form a quadratic equation, which provides extrapolated vehicle speed information for that GPS sensor at t2. Intuitively the reliability of the proposed scheme will decrease as the prediction time duration ( $\mathrm{t} 2-\mathrm{t} 1)$ increases. Considering this factor, measurement noise covariance is increased in the Extended Kalman Filter as the GPS/GPS unsynchronized time (t2-t1 in Fig. 5) increases.

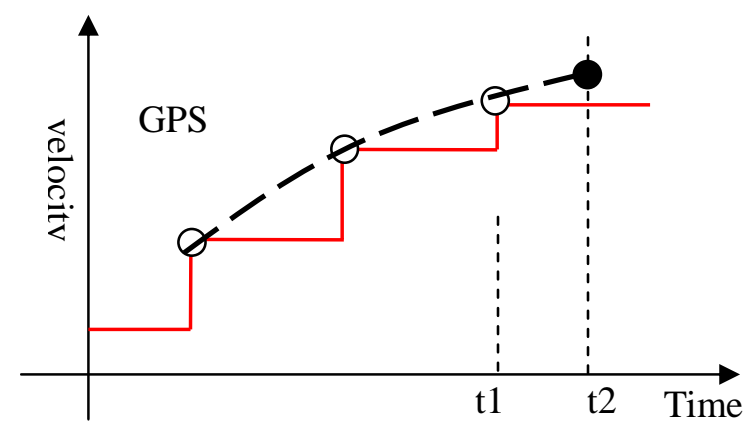

FIGURE 6. THE QUADRATIC EXTRAPOLATION METHOD

\section{EXPERIMENTAL RESULTS}

The overall estimation method combines the vehicle kinematic relationship, an Extended Kalman Filter, and the quadratic extrapolation method for GPS synchronization. To validate the proposed method, a small pick-up truck was equipped with a 2 -axis accelerometer and yaw rate gyro. The 
measurement noise levels $(1 \sigma)$ are $0.02 \mathrm{~m} / \mathrm{s}^{2}$ and $0.08 \mathrm{deg} / \mathrm{s}$ for accelerometers and the rate gyro, respectively. Two GPS receivers (U-blox EVK-5H) were also installed. One unit was located at the front end of the vehicle $\mathrm{x}$-axis and the other was at the rear end of the vehicle $\mathrm{x}$-axis. The distance between the two receivers was $4.8 \mathrm{~m}$. The velocity update rate of the GPS receivers is $2.5 \mathrm{~Hz}$ and the noise levels $(1 \sigma)$ are $0.01 \mathrm{~m} / \mathrm{s}$ for horizontal velocity. The accelerometer/ yaw rate gyro signals were connected to a private CAN bus and GPS signals were hooked to the public CAN bus with high priority. RT2500 from Oxford Technical Solutions was also installed to provide reference signal of the "true" sideslip angle measurement. The J-turn maneuver was conducted on a wet-tile surface ( $\mu=\sim$ 0.1 ). The initial speed was $10 \mathrm{~m} / \mathrm{s}$ and steering angle was greater than $180^{\circ}$. A total of 17 test runs were conducted and test results were collected and processed.

Figure 7 shows example test results. This figure shows the sideslip and heading angle estimation, GPS measurement covariance and the absolute value of yaw rate. Solid lines are estimation results of the proposed method and dashed lines are references from RT2500. The test vehicle was driven straight until $7 \mathrm{sec}$ on high friction surface at a speed of $10 \mathrm{~m} / \mathrm{s}$. Right before entering the low friction area, a large steering angle was applied (around $t=7 \mathrm{sec}$ ). Starting from that point, the vehicle developed a significant sideslip angle. In straight line driving (before $7 \mathrm{sec}$ in Fig. 7), the vehicle yaw rate is low, and as a result, the measurement covariance is high. In Fig. 7-(b), the heading angle estimation does not converge to the reference due to lack of reliable GPS measurement updates. In Fig. 7-(a), the impact of GPS measurement on the sideslip estimation is minimal because the initial sideslip estimation is set to zero and actual sideslip angle with straight line maneuver stays around zero.

After $\mathrm{t}=7 \mathrm{sec}$ in Fig. 7, a near-constant steering angle is applied, resulting in a J-turn with significant yaw rate. Consequently, the measurement covariance stays low. The sideslip and heading angle estimation converged to the reference signals of RT2500. Note that sideslip angle estimation slightly deviates from the reference around $t=12 \mathrm{sec}$, whereas the heading angle estimation stays close to the reference. This can be explained by the observation that the accurate sideslip estimation requires high yaw rate and fast longitudinal speed, where heading angle accuracy only depends on yaw rate (Eq. (10)). A Root Mean Square (RMS) value of sideslip estimation error is $1.93^{\circ}$ (Average RMS results from 17 data set). Since the sideslip estimation accuracy of the proposed method is known to depend on yaw rate and longitudinal velocity, sideslip error is considered only when yaw rate is between $13 \% \mathrm{sec}$ and $17 \% \mathrm{sec}$ and longitudinal speed is in $8 \sim 12 \mathrm{~m} / \mathrm{s}$ range. The calculation assumes that reference signals from RT2500 represent true values. (a) Sideslip Angle

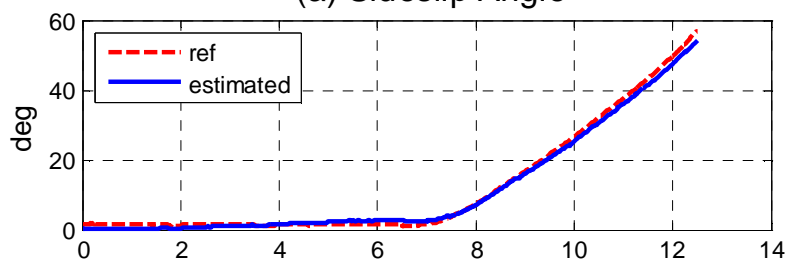

(b) Heading Angle

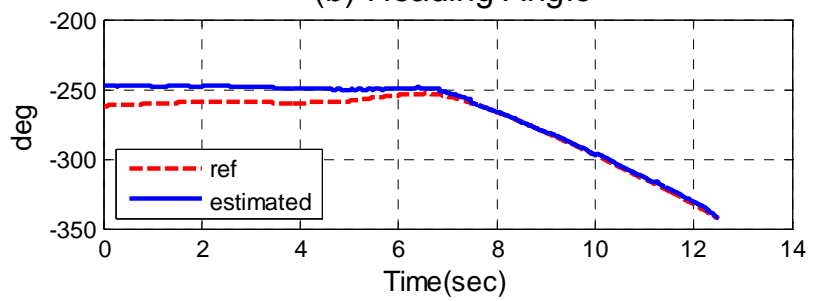

(c) Measurement Covariance

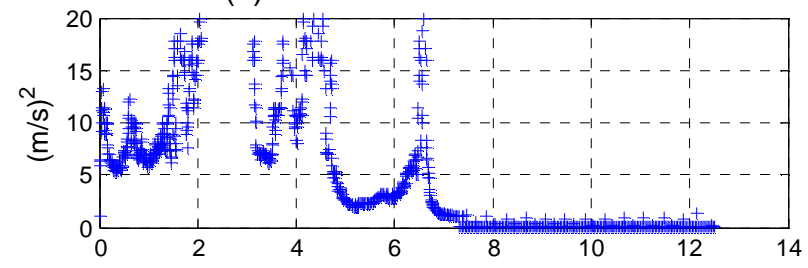

(d) | Yaw Rate |

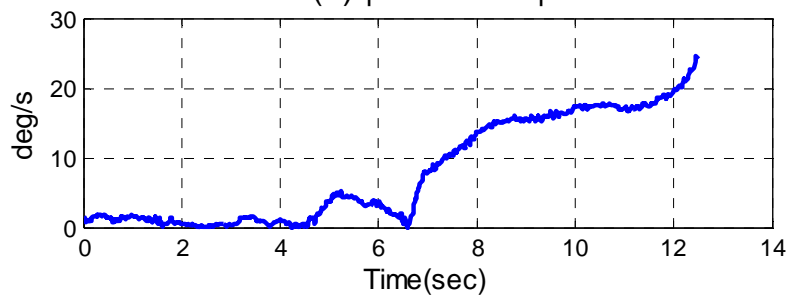

FIGURE 7. (a) SIDESLIP ESTIMATINON (B) HEADING ANGLE ESTIMATION (C) MODIFIED MEASUREMENT COVARIANCE (D) ABSOLUTE VALUE OF YAW RATE

Performance of the GPS/GPS and GPS/IMU synchronization is presented in Fig. 8. It can be seen that if the synchronization among sensors is not taken care of, significant degradation in sideslip angle estimation performance may occur. By considering synchronization, the estimation error is reduced from about 10 degrees to less than 2 degrees when the actual slip angle is 50 degrees (spin out in the J-turn). Clearly, if an active safety system is implemented, the improved side slip angle accuracy helps to trigger the active safety system and maintain proper operation and vehicle control. 


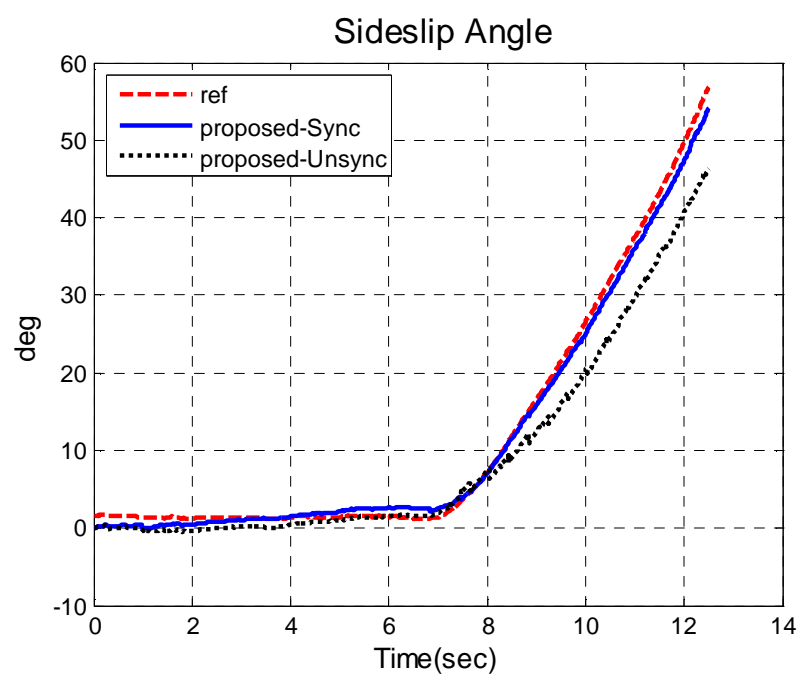

FIGURE 8. THE IMPACT OF GPS/GPS \& GPS/IMU SYNCHRONIZATION ON SIDESLIP ESTIMATION

Next, we investigate the IMU sensor biases and GPS/IMU time latency estimation. There are no reference signals available for comparison. Therefore, it is not possible to assess whether the variables are accurately estimated. Instead, two indirect evaluation methods were used. We check whether the estimation is consistent through all 17 data sets. It is also checked if the proposed method can observe artificially injected bias. Figure 9 shows the bias estimations (longitudinal/lateral acceleration and yaw rate) and GPS/IMU synchronization time estimation for four randomly selected data sets. Consistent trend indicates the proposed method seems to work as intended. Figure 10 shows the lateral accelerometer bias estimation with and without artificial bias (black solid line is without bias, red dash-dot line is with a bias of $0.1 \mathrm{~m} / \mathrm{s}^{2}$ and blue dashed line is with a bias of $-0.1 \mathrm{~m} / \mathrm{s}^{2}$ ). Without the intentionally injected bias, the proposed method yielded a converged value of $0.02 \mathrm{~m} / \mathrm{s}^{2}$ as a bias estimation. However, with bias injection of $0.1 \mathrm{~m} / \mathrm{s}^{2}$ the estimation ended up around $0.05 \mathrm{~m} / \mathrm{s}^{2}$ and it did not converge. The same non-convergence was observed with bias $-0.1 \mathrm{~m} / \mathrm{s}^{2}$ injection. This is because the number of valid GPS data was not substantial enough for the speed of bias estimation. Although the method fails to yield the converged estimation of injected bias, significant increase of bias estimation with $0.1 \mathrm{~m} / \mathrm{s}^{2}$ injection along with decrease caused by $-0.1 \mathrm{~m} / \mathrm{s}^{2}$ injection can infer that bias estimation of the proposed method works.
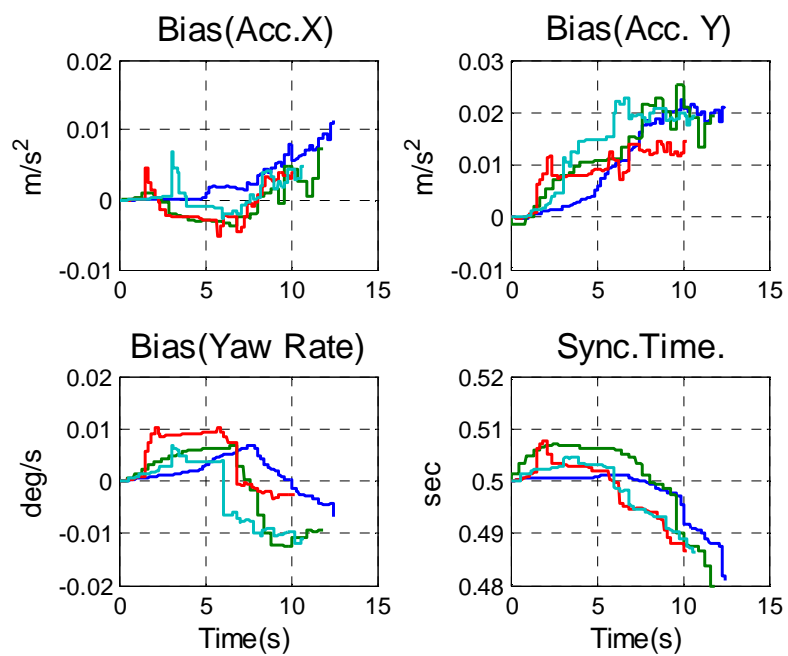

FIGURE 9. VARIOUS STATES ESTIMATIONS FOR RADOMLY SELECTED DATA SET

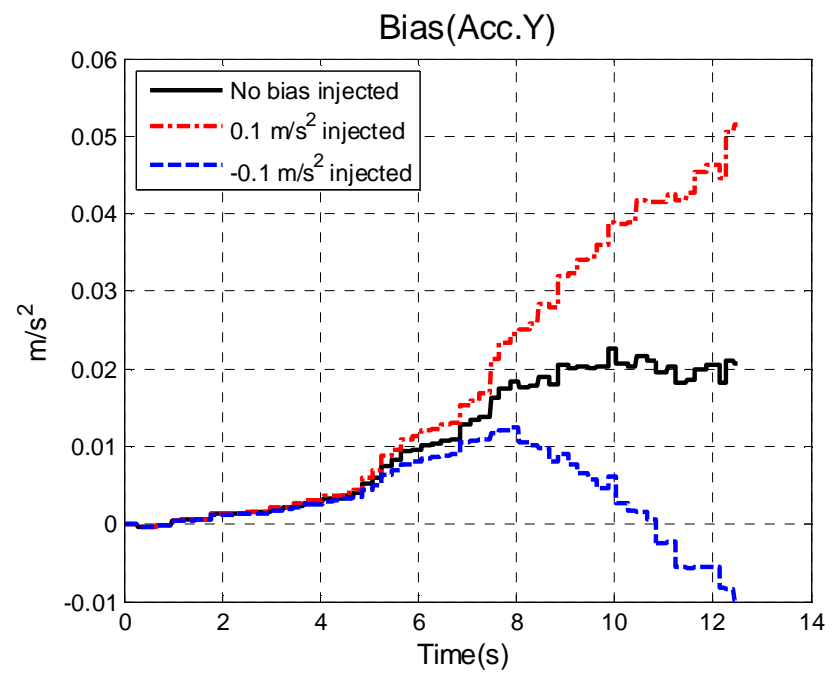

FIGURE 10. THE EFFECT OF INJECTD BIAS ON LATERAL ACCELEROMETER BIAS ESTIMATION.

\section{CONCLUSION}

This paper has shown that the vehicle sideslip angle can be successfully estimated using velocity information from two lowcost GPS receivers. Low update rate of the GPS receiver $(2.5$ $\mathrm{Hz}$ ) was compensated by fast sampling IMU through an Extended Kalman Filter. In addition, the IMU sensor biases were estimated. A software method was successfully employed for GPS/IMU synchronization. Asynchronous updates from the two GPS receivers were found to be a source of estimation error and a quadratic extrapolation method was used to reduce its negative impact.

Our analysis indicated that the sideslip estimation accuracy of the proposed method depends on the distance between the 
two GPS receivers, the accuracy of the GPS speed error, vehicle yaw rate and forward speed. Assuming the two GPS receivers are installed on the front and rear ends of the vehicle, with today's GPS velocity error of $0.01 \mathrm{~m} / \mathrm{s}$ (standard deviation), the predicted accuracy $(1 \sigma)$ is around $0.65^{\circ}$ when vehicle speed is $10 \mathrm{~m} / \mathrm{sec}$ and yaw rate is $15 \mathrm{deg} / \mathrm{sec}$ (which is selected as a typical sliding on icy scenario). However, our experimental results have a much higher value of around $1.93^{\circ}$. The unsynchronized GPS/GPS and GPS/IMU updates are believed to be major error sources even after attenuation.

It has been confirmed that the proposed method based on GPS measurements cannot observe the sideslip when yaw rate is zero or longitudinal velocity is very low. However, those conditions correspond to safe driving situation, which does not require sideslip angle estimation. One exception is excessive side sliding. However, since side sliding is typically followed by significant yaw rate, the proposed method can provide reliable sideslip information after yaw rate starts to build up.

Although this paper utilized two GPS receivers, it is lowcost compared to existing dual-antenna GPS kit for sideslip estimation. It is because this work uses only velocity measurement, whereas the later uses phase difference of carrier waves. The GPS receiver/development kit we chose to evaluate is among the cheaper ones $(\$ 100)$ to prove the feasibility of the proposed method.

\section{ACKNOWLEDGMENTS}

The authors would like to thank TRW Automotive for providing test vehicle and sensors. They also would like to acknowledge Kevin Fontenot and Jeremy Reeve of TRW Automotive for their time and technical contribution for the experimental work.

\section{REFERENCES}

[1] Nishio, A., and Tozu, K., 2001, "Development of Vehicle Stability Control System Based on Vehicle Sideslip Angle Estimation," New York.

[2] Li, L., Wang, F.-Y., and Zhou, Q., 2005, "A Robust Observer Designed for Vehicle Lateral Motion Estimation," Proc. IEEE proceedings on Intelligent Vehicle Symposium, pp. 417-422.

[3] Gao, X., and Yu, Z., 2008, "Vehicle Sideslip Angel Estimation by Using High Gain Observer," Proc. AVEC 9th International Symposium on Advanced Vehicle Control, Kobe, Japan.

[4] Kiencke, U., and Daiss, A., 1997, "Observation of Lateral Vehicle Dynamics," Control Engineering Practice, 5(8), pp. 1145-1150.

[5] Piyabongkarn, D., Rajamani, R., Grogg, J. A., and Lew, J. Y., 2006, "Development and Experimental Evaluation of a Slip Angle Estimator for Vehicle Stability Control," Proc. Proceeding of the 2006 American Control Conference, Minneapolis, Minnesota.
[6] Yih, P., Ryu, J., and Gerdes, J. C., 2004, "Vehicle State Estimation Using Steering Torque," Proc. Proceeding of the 2004 American Control Conference, Boston, Massachusetts.

[7] Anderson, R., and Bevly, D. M., 2004, "Estimation of Slip Angles Using a Model Based Estimator and Gps," Proc. Proceeding of the 2004 American Control Conference, Boston, Massachusetts.

[8] Bevly, D. M., 2004, "Global Positioning System (Gps): A Low-Cost Velocity Sensor for Correcdting Inertial Sensor Errors on Ground Vehicles," Journal of Dynamic System, Measurement, and Control, 126(pp. 255-264.

[9] Bevly, D. M., Gerdes, J. C., and Wilson, C., 2003, "The Use of Gps Based Velocity Measurements for Measurement of Sideslip and Wheel Slip," Vehicle System Dynamics, 38(2), pp. 127-147.

[10] Ryu, J., Rossetter, E. J., and Gerdes, J. C., 2002, "Vehicle Sideslip and Roll Parameter Estimation Using Gps," Proc. AVEC 6th International Symposium on Advanced Vehicle Control, Hiroshima,Japan.

[11] Hrovat, D., Tseng, H. E., and Yester, J. L., 2003, "Vehicle Dynamic Measuring Apparatus and Method Using Multiple Gps Antennas," Ford Motor Company, USA.

[12] Ryu, J., 2004, "State and Parameter Estimation for Vehicle Dynamics Control Using Gps," Doctor of Philosophy Stanford University.

[13] Li, B., Rizos, C., Lee, H. K., and Lee, H. K., 2006, "A Gps-Slaved Time Synchronization System for Hybrid Navigation," GPS Solutions, 10(pp. 207-217.

[14] Skog, I., and Handel, P., 2008, "Effects of Time Synchronization Errors in Gnss-Aided Ins," Proc. Proceedings of Position, Location and Navigation Symposium, Monterey, California.

[15] Stengel, R., 1994, Optimal Control and Estimation, Dover Publications, Inc., New York.

[16] Franklin, G. F., Powell, J. D., and Workman, M., 1998, Digital Control of Dynamic Systems, Addison Wesley Longman, Inc., Menlo Park, CA. 